\title{
EISENSTEIN POLYNOMIALS OVER FUNCTION FIELDS
}

\author{
EDOARDO DOTTI AND GIACOMO MICHELI
}

\begin{abstract}
In this paper we compute the density of monic and non-monic Eisenstein polynomials of fixed degree having entries in an integrally closed subring of a function field over a finite field.
\end{abstract}

Keywords: Function fields, Density, Polynomials, Riemann-Roch spaces.

MSC: 11R58, 11T06

\section{INTRODUCTION}

Let us start with the definition of Eisenstein polynomial and natural density

Definition 1. Let $R$ be an integral domain. A polynomial $f(X)=\sum_{i=0}^{n} a_{i} x^{i} \in R[X]$ is said to be Eisenstein if there exists a prime ideal $\mathfrak{p} \subseteq R$ for which

- $a_{i} \in \mathfrak{p}$ for all $i \in\{0, \ldots, n-1\}$,

- $a_{0} \notin \mathfrak{p}^{2}$,

- $a_{n} \notin \mathfrak{p}$.

Definition 2. A subset $A$ of $\mathbb{Z}^{n}$ is said to have density $a$ if

$$
a=\lim _{B \rightarrow \infty} \frac{\mid A \cap\left[-B, B\left[^{n} \mid\right.\right.}{(2 B)^{n}} .
$$

A classical result from the literature is that any Eisenstein polynomial is irreducible. In addition, observe that any polynomial of degree at most $d$ and coefficients over $\mathbb{Z}$ can be regarded as an element of $\mathbb{Z}^{d+1}$, while any monic polynomial of degree $d$ can be regarded as an element of $\mathbb{Z}^{d}$. Recently, it has been of interest the explicit computation of the natural density of both degree $d$ Eisenstein polynomials and monic Eisenstein polynomials over $\mathbb{Z}$, see for example $[3,1]$.

As was first proved by Dubickas in [1], the natural density of monic Eisenstein polynomials over $\mathbb{Z}$ of fixed degree $d$ is

$$
\prod_{p \text { prime }}\left(1-\frac{p-1}{p^{d+1}}\right) .
$$

Heyman and Shparlinski extended the results of Dubickas to general Eisenstein polynomials and computed the error term of the density [3, Theorem 1, Theorem 2].

The second author was partially supported by Swiss National Science Foundation grant number 149716 and Armasuisse. 
In this paper we would like to establish a function field analogue of these results that will include all the cases in which $R$ is selected as an integrally closed subring of a function field of a curve over a finite field.

The general case that we will analyse needs an appropriate definition of density which makes use of Moore-Smith convergence for directed sets, as described in [5]. For the moment, let us fix the notation for the basic structures we are are going to deal with, which is essentially the same as in [6].

Let $q$ be a prime power and $\mathbb{F}_{q}$ be the finite field of order $q$. Let $F$ be a function field having full constant field $\mathbb{F}_{q}$. Let $\mathbb{P}_{F}$ be the set of places of $F$ and $\mathcal{S}$ a non empty proper subset of $\mathbb{P}_{F}$. Let us denote by $\mathcal{O}_{P}$ the valuation ring at a place $P$ of $F$. Let $H=\bigcap_{P \in \mathcal{S}} \mathcal{O}_{P}$ be the holomorphy ring associated to $\mathcal{S}$ [6, Definition 3.2.2]. As it is well known, $H$ is a Dedekind Domain therefore any prime ideal is also maximal. In addition the maximal ideals of $H$ correspond exactly to the places in $\mathcal{S}$ see [6, Proposition 3.2.9]. Therefore, if $P$ is a place of $F$ which lies in $\mathcal{S}$ there exists a unique maximal ideal $P_{H} \subseteq H$ corresponding to $P$ for which $P \cap H=P_{H}$. In order not to heavier the notation, we will denote $P_{H}$ again by $P$. Let $\mathcal{D}$ be the set of positive divisors of $\operatorname{Div}(F)$ having support outside the holomorphy set $\mathcal{S}$. It is easy to observe

$$
H=\bigcup_{D \in \mathcal{D}} \mathcal{L}(D)
$$

and that $\mathcal{D}$ is a directed set.

Let now $A \subseteq H^{m}$, we define the upper and lower density of $A$ as

$$
\begin{aligned}
& \overline{\mathbb{D}}(A)=\limsup _{D \in \mathcal{D}} \frac{\left|A \cap \mathcal{L}(D)^{m}\right|}{q^{m \ell(D)}}, \\
& \underline{\mathbb{D}}(A)=\liminf _{D \in \mathcal{D}} \frac{\left|A \cap \mathcal{L}(D)^{m}\right|}{q^{m \ell(D)}}
\end{aligned}
$$

where the limit is defined using Moore-Smith convergence over the directed set $\mathcal{D}$ (see $[4$, Chapter 2]). The density of $A$ is then defined if $\underline{\mathbb{D}}(A)=\overline{\mathbb{D}}(A)=: \mathbb{D}(A)$.

As already observed in [5], if we specialize our definition of density to the case of the univariate polynomial ring over a finite field we get the usual definition of density for $\mathbb{F}_{q}[x]$, see for example in $[2,7]$.

In addition, the final formulas for the density we get are analogous to the ones over the rational integers obtained in $[1,3]$.

The paper is structured as follows: in the next subsection we specify the notation we are going to use for the rest of the paper, in section 2 we compute the density of monic Eisenstein polynomials, in section 3 we apply a similar strategy to compute the density of general Eisenstein polynomials.

1.1. Notation. Throughout this paper, when $Y$ is a set and $m$ is a positive integer, we will denote by $Y^{m}$ the cartesian product of $m$-copies of $Y$. To avoid confusion, the square of an ideal $Q$ will then be denoted by $\widehat{Q}=Q \cdot Q$. Furthermore notice that in 
the whole paper we consider polynomials of degree $d>1$. To easier the notation, we fix an enumeration $\left\{Q_{1}, Q_{2}, \ldots, Q_{i}, \ldots\right\}$ of the places of $\mathcal{S}$. Since we will deal with the density of both monic and non-monic Eisenstein polynomials, we have to distinguish the notation, which we clarify in the following two paragraphs.

Notation for monic Eisenstein polynomials: with a small abuse of notation we identify $H^{d}$ with the set of all monic polynomials of degree $d$ having entries over $H$. In particular, if $\left(h_{0}, \ldots, h_{d-1}\right) \in H^{d}$ then $h_{i}$ denotes the coefficient of the monomial of degree $i$. Furthermore, we denote by $\mathcal{E} \subset H^{d}$ the set of monic Eisenstein polynomials of degree $d$ and by $\mathcal{N}$ its complement in $H^{d}$. We denote by $\mathcal{E}_{i}$ the set of monic polynomials which are Eisenstein with respect to $Q_{i}$ :

$$
\mathcal{E}_{i}=\left\{\left(h_{0}, \ldots, h_{d-1}\right) \in H^{d}: \quad h_{i} \in Q_{i} \forall i \in\{0, \ldots d-1\} \text { and } h_{0} \notin \widehat{Q}_{i}\right\} .
$$

We denote by $\mathcal{N}_{i}$ the complement of $\mathcal{E}_{i}$.

Notation for Eisenstein polynomials: Analogously, we identify the set of all polynomials of degree $d$ having entries over $H$ with $H^{d+1}$. Let $\mathcal{E}^{+} \subseteq H^{d+1}$ be the set of Eisenstein polynomials of degree $d$ and $\mathcal{N}^{+}$be its complement in $H^{d+1}$. We denote by $\mathcal{E}_{i}^{+}$the set of polynomials which are Eisenstein with respect to $Q_{i}$ :

$$
\mathcal{E}_{i}^{+}=\left\{\left(h_{0}, \ldots, h_{d}\right) \in H^{d+1}: \quad h_{i} \in Q_{i} \forall i \in\{0, \ldots d-1\}, h_{0} \notin \widehat{Q}_{i} \text { and } h_{d} \notin Q_{i}\right\} .
$$

We denote by $\mathcal{N}_{i}^{+}$the complement of $\mathcal{E}_{i}^{+}$.

\section{Monic Eisenstein Polynomials}

In this section we compute the density of monic Eisenstein polynomials via approximating the complement of $\mathcal{E}$ (i.e. $\mathcal{N}$ ) with $\overline{\mathcal{N}_{t}}=\bigcap_{i=1}^{t} \mathcal{N}_{i}$. First we show that we can explicitly compute the density of $\overline{\mathcal{N}_{t}}$ (Proposition 3 ). Then, we give a criterion to check whether the approximation is "sharp": i.e. whether the limit of the densities of $\overline{\mathcal{N}_{t}}$ converges to the density of $\mathcal{N}$ (Lemma 4). Finally, we verify that the conditions under which the approximation is sharp are verified (Theorem 5).

Proposition 3. The density of $\overline{\mathcal{N}_{t}}$ is

$$
\mathbb{D}\left(\overline{\mathcal{N}_{t}}\right)=\prod_{i=1}^{t}\left(1-\frac{q^{\operatorname{deg}\left(Q_{i}\right)}-1}{q^{(d+1) \operatorname{deg}\left(Q_{i}\right)}}\right) .
$$

Proof. Consider the map

$$
\tilde{\phi}: H^{d} \rightarrow\left(H /\left(\widehat{Q}_{1} \cdots \widehat{Q}_{t}\right)\right)^{d},
$$

which is defined componentwise by the reduction modulo the ideal $\left(\widehat{Q}_{1} \cdots \widehat{Q}_{t}\right)$. Observe also that $\left(H /\left(\widehat{Q}_{1} \cdots \widehat{Q}_{t}\right)\right)^{d} \simeq \prod_{i=1}^{t}\left(H / \widehat{Q}_{i}\right)^{d}$ by the Chinese Remainder Theorem.

Consider now a divisor $D \in \mathcal{D}$. In order to compute the density of $\overline{\mathcal{N}_{t}}$ it is enough to count how many elements there are in $\overline{\mathcal{N}_{t}} \cap \mathcal{L}(D)^{d}$, when the degree of $D$ is large. 
We start by showing that $\mathcal{L}(D)^{d}$ maps surjectively onto $\left(H /\left(\widehat{Q}_{1} \cdots \widehat{Q}_{t}\right)\right)^{d}$ when the degree of $D$ is large enough.

For this consider the $\mathbb{F}_{q}$ linear map $\phi: \mathcal{L}(D) \rightarrow\left(H /\left(\widehat{Q}_{1} \cdots \widehat{Q}_{t}\right)\right)$. We have $\operatorname{ker}(\phi)=$ $\mathcal{L}(D) \cap\left(\widehat{Q}_{1} \cdots \widehat{Q}_{t}\right)$, which represents the elements of $\mathcal{L}(D)$ having at least a double root at each $Q_{i}$. Hence $\operatorname{ker}(\phi)=\mathcal{L}\left(D-2 \sum_{i=1}^{t} Q_{i}\right)$.

By Riemann's theorem [6, Theorem 1.4.17], if the degree of $D$ is large enough, the dimension of the kernel as an $\mathbb{F}_{q}$ vector space is

(2) $\ell\left(D-2 \sum_{i=1}^{t} Q_{i}\right)=\operatorname{deg}\left(D-2 \sum_{i=1}^{t} Q_{i}\right)+1-g=\operatorname{deg}(D)-2 \sum_{i=1}^{t} \operatorname{deg}\left(Q_{i}\right)+1-g$,

where $g$ denotes the genus of the function field.

By the same theorem $\ell(D)=\operatorname{deg}(D)+1-g$. Hence we obtain

$$
\operatorname{dim}_{\mathbb{F}_{q}}(\mathcal{L}(D) / \operatorname{ker}(\phi))=\ell(D)-\ell\left(D-2 \sum_{i=1}^{t} Q_{i}\right)=2 \sum_{i=1}^{t} \operatorname{deg}\left(Q_{i}\right) .
$$

On the other hand, by the Chinese Remainder Theorem

$$
\operatorname{dim}_{\mathbb{F}_{q}}\left(H /\left(\widehat{Q}_{1} \cdots \widehat{Q}_{t}\right)\right) \stackrel{C}{=} T \operatorname{dim}_{\mathbb{F}_{q}}\left(H / \widehat{Q}_{1} \times \cdots \times H / \widehat{Q}_{t}\right)=2 \sum_{i=1}^{t} \operatorname{deg}\left(Q_{i}\right) .
$$

Therefore when the degree of $D$ is large enough $\phi$ is surjective, thus $\tilde{\phi}$ is surjective.

Let $\psi_{i}:\left(H /\left(\widehat{Q}_{1} \cdots \widehat{Q}_{t}\right)\right)^{d} \longrightarrow\left(H / \widehat{Q}_{i}\right)^{d}$. We have the following situation:

$$
\mathcal{L}(D)^{d} \stackrel{\tilde{\phi}}{\rightarrow}\left(H /\left(\widehat{Q}_{1} \cdots \widehat{Q}_{t}\right)\right)^{d} \stackrel{\psi}{\rightarrow} \prod_{i=1}^{t}\left(H / \widehat{Q}_{i}\right)^{d}
$$

where $\psi=\left(\psi_{1}, \ldots, \psi_{t}\right)$. Notice that the check for $f \in H^{d}$ not to be Eisenstein with respect to $Q_{i}$ can be performed by looking at the reduction modulo $\widehat{Q}_{i}$. Therefore $f \in \overline{\mathcal{N}_{t}} \cap \mathcal{L}(D)^{d}$ if and only if $\psi_{i} \circ \tilde{\phi}(f) \notin\left(\left(Q_{i} / \widehat{Q}_{i}\right) \backslash\{0\}\right) \times\left(Q_{i} / \widehat{Q}_{i}\right)^{d-1}=: E_{i}$ for all $i \in\{1, \ldots, t\}$.

It follows that $\overline{\mathcal{N}_{t}} \cap \mathcal{L}(D)^{d}=\tilde{\phi}^{-1}\left(\psi^{-1}\left(\prod_{i=1}^{t}\left(\left(H / \widehat{Q}_{i}\right)^{d} \backslash E_{i}\right)\right)\right) \cap \mathcal{L}(D)^{d}$. Hence

$$
\begin{aligned}
\left|\overline{\mathcal{N}_{t}} \cap \mathcal{L}(D)^{d}\right| & =|\operatorname{ker}(\tilde{\phi})| \cdot \prod_{i=1}^{t}\left|\left(H / \widehat{Q}_{i}\right)^{d} \backslash E_{i}\right| \\
& =q^{d\left(\operatorname{deg}(D)-2 \sum_{i=1}^{t} \operatorname{deg}\left(Q_{i}\right)+1-g\right)} \cdot \prod_{i=1}^{t}\left|\left(H / \widehat{Q}_{i}\right)^{d} \backslash E_{i}\right|,
\end{aligned}
$$

where the last equality follows from (2). Now it remains to compute

$$
\begin{aligned}
\left|\left(H / \widehat{Q}_{i}\right)^{d} \backslash E_{i}\right| & =q^{2 d \operatorname{deg}\left(Q_{i}\right)}-\left|\left(\left(Q_{i} / \widehat{Q}_{i}\right) \backslash\{0\}\right) \times\left(Q_{i} / \widehat{Q}_{i}\right)^{d-1}\right| \\
& =q^{2 d \operatorname{deg}\left(Q_{i}\right)}-\left(q^{\operatorname{deg}\left(Q_{i}\right)}-1\right) \cdot q^{(d-1) \operatorname{deg}\left(Q_{i}\right)} \\
& =q^{2 d \operatorname{deg}\left(Q_{i}\right)}\left(1-q^{-d \operatorname{deg}\left(Q_{i}\right)}+q^{-(d+1) \operatorname{deg}\left(Q_{i}\right)}\right) .
\end{aligned}
$$


Therefore for $D$ of degree large enough

$$
\begin{gathered}
\frac{\left|\overline{\mathcal{N}_{t}} \cap \mathcal{L}(D)^{d}\right|}{\left|\mathcal{L}(D)^{d}\right|}=\frac{q^{d\left(\operatorname{deg}(D)-2 \sum_{i=1}^{t} \operatorname{deg}\left(Q_{i}\right)+1-g\right)}}{q^{d(\operatorname{deg}(D)+1-g)}} \cdot \prod_{i=1}^{t} q^{2 d \operatorname{deg}\left(Q_{i}\right)}\left(1-q^{-d \operatorname{deg}\left(Q_{i}\right)}+q^{-(d+1) \operatorname{deg}\left(Q_{i}\right)}\right) \\
\quad=\prod_{i=1}^{t}\left(1-q^{-d \operatorname{deg}\left(Q_{i}\right)}+q^{-(d+1) \operatorname{deg}\left(Q_{i}\right)}\right)=\prod_{i=1}^{t}\left(1-\frac{q^{\operatorname{deg}\left(Q_{i}\right)}-1}{q^{(d+1) \operatorname{deg}\left(Q_{i}\right)}}\right) .
\end{gathered}
$$

Hence

$$
\mathbb{D}\left(\overline{\mathcal{N}_{t}}\right)=\lim _{D \in \mathcal{D}} \frac{\left|\overline{\mathcal{N}_{t}} \cap \mathcal{L}(D)^{d}\right|}{\left|\mathcal{L}(D)^{d}\right|}=\prod_{i=1}^{t}\left(1-\frac{q^{\operatorname{deg}\left(Q_{i}\right)}-1}{q^{(d+1) \operatorname{deg}\left(Q_{i}\right)}}\right)
$$

Lemma 4. Let $n \in \mathbb{N}, A \subseteq H^{n}$. Let $\left\{A_{t}\right\}_{t \in \mathbb{N}}$ be a family of subsets of $H^{n}$ such that $A_{t+1} \subseteq A_{t}$ and $\bigcap_{t \in \mathbb{N}} A_{t}=A$. Assume also that $\mathbb{D}\left(A_{t}\right)$ exists for all $t$. If $\lim _{t \rightarrow \infty} \overline{\mathbb{D}}\left(A_{t} \backslash\right.$ $A)=0$, then $\mathbb{D}(A)=\lim _{t \rightarrow \infty} \mathbb{D}\left(A_{t}\right)$.

Proof. We start from the equality $\left|A_{t} \cap \mathcal{L}(D)^{n}\right|=\left|A \cap \mathcal{L}(D)^{n}\right|+\left|\left(A_{t} \backslash A\right) \cap \mathcal{L}(D)^{n}\right|$, from which it follows

$$
\begin{aligned}
\liminf _{D \in \mathcal{D}} \frac{\left|A \cap \mathcal{L}(D)^{n}\right|}{\left|\mathcal{L}(D)^{n}\right|} & =\liminf _{D \in \mathcal{D}}\left(\frac{\left|A_{t} \cap \mathcal{L}(D)^{n}\right|}{\left|\mathcal{L}(D)^{n}\right|}-\frac{\left|\left(A_{t} \backslash A\right) \cap \mathcal{L}(D)^{n}\right|}{\left|\mathcal{L}(D)^{n}\right|}\right) \\
& \geq \liminf _{D \in \mathcal{D}} \frac{\left|A_{t} \cap \mathcal{L}(D)^{n}\right|}{\left|\mathcal{L}(D)^{n}\right|}-\limsup _{D \in \mathcal{D}} \frac{\left|\left(A_{t} \backslash A\right) \cap \mathcal{L}(D)^{n}\right|}{\left|\mathcal{L}(D)^{n}\right|} .
\end{aligned}
$$

It follows that $\underline{\mathbb{D}}\left(A_{t}\right)-\overline{\mathbb{D}}\left(A_{t} \backslash A\right) \leq \underline{\mathbb{D}}(A)$. Since $\mathbb{D}\left(A_{t}\right)$ exists for all $t$ we get

$$
\mathbb{D}\left(A_{t}\right)-\overline{\mathbb{D}}\left(A_{t} \backslash A\right) \leq \underline{\mathbb{D}}(A) .
$$

Now notice that $\lim _{t \rightarrow \infty} \mathbb{D}\left(A_{t}\right)$ exists since $\mathbb{D}\left(A_{t}\right)$ is decreasing and bounded from below. By taking the limit in $t$, the last expression then becomes

$$
\lim _{t \rightarrow \infty} \mathbb{D}\left(A_{t}\right)-\lim _{t \rightarrow \infty} \overline{\mathbb{D}}\left(A_{t} \backslash A\right) \leq \underline{\mathbb{D}}(A)
$$

Since $\lim _{t \rightarrow \infty} \overline{\mathbb{D}}\left(A_{t} \backslash A\right)=0$ by assumption, it follows that $\lim _{t \rightarrow \infty} \mathbb{D}\left(A_{t}\right) \leq \underline{\mathbb{D}}(A)$.

On the other hand $A \subseteq A_{t}$ which implies $\overline{\mathbb{D}}(A) \leq \mathbb{D}\left(A_{t}\right)$. In particular $\overline{\mathbb{D}}(A) \leq$ $\lim _{t \rightarrow \infty} \mathbb{D}\left(A_{t}\right)$. Combining all together we get

$$
\lim _{t \rightarrow \infty} \mathbb{D}\left(A_{t}\right) \leq \underline{\mathbb{D}}(A) \leq \overline{\mathbb{D}}(A) \leq \lim _{t \rightarrow \infty} \mathbb{D}\left(A_{t}\right),
$$

therefore the claim follows.

Theorem 5. The density of the set of monic Eisenstein polynomials with coefficients in $H$ is

$$
\mathbb{D}(\mathcal{E})=1-\prod_{Q \in \mathcal{S}}\left(1-\frac{q^{\operatorname{deg}(Q)}-1}{q^{(d+1) \operatorname{deg}(Q)}}\right) .
$$


Proof. We make use of Lemma 4 for the family $\left\{\overline{\mathcal{N}_{t}}\right\}_{t \in \mathbb{N}}$. Hence we want to show that $\lim _{t \rightarrow \infty} \overline{\mathbb{D}}\left(\overline{\mathcal{N}_{t}} \backslash \mathcal{N}\right)=0$.

First note that

- $\overline{\mathcal{N}_{t}} \backslash \mathcal{N}=\bigcup_{r>t} \mathcal{E}_{r} \subseteq \bigcup_{r>t} Q_{r}^{d}$,

- $Q_{r}^{d} \cap \mathcal{L}(D)^{d}=\mathcal{L}\left(D-Q_{r}\right)=0$, if $\operatorname{deg}(D)-\operatorname{deg}\left(Q_{r}\right)<0$.

Now we get

$$
\begin{aligned}
& \overline{\mathbb{D}}\left(\overline{\mathcal{N}_{t}} \backslash \mathcal{N}\right)=\limsup _{D \in \mathcal{D}} \frac{\left|\left(\overline{\mathcal{N}_{t}} \backslash \mathcal{N}\right) \cap \mathcal{L}(D)^{d}\right|}{\left|\mathcal{L}(D)^{d}\right|} \leq \limsup _{D \in \mathcal{D}}\left|\bigcup_{\substack{r>t \\
\operatorname{deg}\left(Q_{r}\right) \leq \operatorname{deg}(D)}} Q_{r}^{d} \cap \mathcal{L}(D)^{d}\right| q^{-d \ell(D)} \\
& (3) \quad=\limsup _{D \in \mathcal{D}}\left|\bigcup_{\substack{r>t \\
\operatorname{deg}\left(Q_{r}\right) \leq \operatorname{deg}(D)}} \mathcal{L}\left(D-Q_{r}\right)^{d}\right| q^{-d \ell(D)} \leq \limsup _{D \in \mathcal{D}} \sum_{\substack{r>t \\
\operatorname{deg}\left(Q_{r}\right) \leq \operatorname{deg}(D)}} \frac{q^{d \ell\left(D-Q_{r}\right)}}{q^{d \ell(D)}} .
\end{aligned}
$$

Observe now that if $\operatorname{deg}\left(D-Q_{r}\right) \geq 0$ we have that $\ell\left(D-Q_{r}\right) \leq \operatorname{deg}\left(D-Q_{r}\right)+1[6$, Eq. 1.21] and also that $\ell(D) \geq \operatorname{deg}(D)+1-g$ by Riemann's theorem.

Hence we have that (3) is less or equal than

$$
\limsup _{D \in \mathcal{D}} \sum_{\substack{r>t \\ \operatorname{deg}\left(Q_{r}\right) \leq \operatorname{deg}(D)}} \frac{q^{d\left(1+\operatorname{deg}(D)-\operatorname{deg}\left(Q_{r}\right)\right)}}{q^{d(\operatorname{deg}(D)+1-g)}} \leq \sum_{r>t} q^{d\left(g-\operatorname{deg}\left(Q_{r}\right)\right)}=q^{d g} \sum_{r>t} q^{-d \operatorname{deg}\left(Q_{r}\right)} .
$$

We now notice that $\sum_{r>t} q^{-d \operatorname{deg}\left(Q_{r}\right)}$ is the tail of a subseries of the Zeta function, which is absolutely convergent for $d>1$. Letting $t$ going to infinity the tail converges to 0 , thus $\lim _{t \rightarrow \infty} \overline{\mathbb{D}}\left(\overline{\mathcal{N}_{t}} \backslash \mathcal{N}\right)=0$. We are now able to apply Lemma 4 with $n=d, A_{t}=\overline{\mathcal{N}_{t}}$ and $A=\mathcal{N}$

$$
\mathbb{D}(\mathcal{N})=\lim _{t \rightarrow \infty} \mathbb{D}\left(\overline{\mathcal{N}_{t}}\right)=\lim _{t \rightarrow \infty} \prod_{i=1}^{t}\left(1-\frac{q^{\operatorname{deg}\left(Q_{i}\right)}-1}{q^{(d+1) \operatorname{deg}\left(Q_{i}\right)}}\right)=\prod_{Q \in \mathcal{S}}\left(1-\frac{q^{\operatorname{deg}(Q)}-1}{q^{(d+1) \operatorname{deg}(Q)}}\right) .
$$

We conclude by taking the complement

$$
\mathbb{D}(\mathcal{E})=1-\mathbb{D}(\mathcal{N})=1-\prod_{Q \in \mathcal{S}}\left(1-\frac{q^{\operatorname{deg}(Q)}-1}{q^{(d+1) \operatorname{deg}(Q)}}\right) .
$$

\section{Non-Monic Eisenstein Polynomials}

In this section we compute the density of Eisenstein polynomials applying the same strategy of section 2. For this let $\overline{\mathcal{N}}_{t}^{+}=\bigcap_{i=1}^{t} \mathcal{N}_{i}^{+}$.

Proposition 6. The density of $\overline{\mathcal{N}}^{+}$is

$$
\mathbb{D}\left(\overline{\mathcal{N}}_{t}^{+}\right)=\prod_{i=1}^{t}\left(1-\frac{\left(q^{\operatorname{deg}\left(Q_{i}\right)}-1\right)^{2}}{q^{(d+2) \operatorname{deg}\left(Q_{i}\right)}}\right) .
$$


Proof. Consider a divisor $D \in \mathcal{D}$. With the same reasoning of the monic case one can show that $\mathcal{L}(D)^{d+1}$ maps surjectively onto $\left(H /\left(\widehat{Q}_{1} \cdots \widehat{Q}_{t}\right)\right)^{d+1}$ when the degree of $D$ is large enough.

Let $\psi_{i}:\left(H /\left(\widehat{Q}_{1} \cdots \widehat{Q}_{t}\right)\right)^{d+1} \longrightarrow\left(H / \widehat{Q}_{i}\right)^{d+1}$ as before. The situation is now the following:

$$
\mathcal{L}(D)^{d+1} \stackrel{\tilde{\phi}}{\rightarrow}\left(H /\left(\widehat{Q}_{1} \cdots \widehat{Q}_{t}\right)\right)^{d+1} \stackrel{\psi}{\rightarrow} \prod_{i=1}^{t}\left(H / \widehat{Q}_{i}\right)^{d+1}
$$

where $\psi=\left(\psi_{1}, \ldots, \psi_{t}\right)$.

Analogously to the case of monic polynomials we note that we can verify that $f \in H$ is not Eisenstein with respect to $Q_{i}$ by looking at the reduction modulo $\widehat{Q}_{i}$. Hence $f \in \overline{\mathcal{N}}_{t}^{+} \cap$ $\mathcal{L}(D)^{d+1}$ if and only if $\psi_{i} \circ \tilde{\phi}(f) \notin\left(\left(Q_{i} / \widehat{Q}_{i}\right) \backslash\{0\}\right) \times\left(Q_{i} / \widehat{Q}_{i}\right)^{d-1} \times\left(\left(H / \widehat{Q}_{i}\right) \backslash\left(Q_{i} / \widehat{Q}_{i}\right)\right)=$ : $E_{i}^{+}$for all $i \in\{1, \ldots, t\}$.

Hence we get

$$
\begin{aligned}
& \left|\overline{\mathcal{N}}_{t}^{+} \cap \mathcal{L}(D)^{d+1}\right|=|\operatorname{ker}(\tilde{\phi})| \cdot \prod_{i=1}^{t}\left|\left(H / \widehat{Q}_{i}\right)^{d+1} \backslash E_{i}^{+}\right| \\
= & q^{(d+1)\left(\operatorname{deg}(D)-2 \sum_{i=1}^{t} \operatorname{deg}\left(Q_{i}\right)+1-g\right)} \cdot \prod_{i=1}^{t}\left|\left(H / \widehat{Q}_{i}\right)^{d+1} \backslash E_{i}^{+}\right|,
\end{aligned}
$$

where

$$
\begin{aligned}
\left|\left(H / \widehat{Q}_{i}\right)^{d+1} \backslash E_{i}^{+}\right| & =q^{2(d+1) \operatorname{deg}\left(Q_{i}\right)}-\left|\left(\left(Q_{i} / \widehat{Q}_{i}\right) \backslash\{0\}\right) \times\left(Q_{i} / \widehat{Q}_{i}\right)^{d-1} \times\left(\left(H / \widehat{Q}_{i}\right) \backslash\left(Q_{i} / \widehat{Q}_{i}\right)\right)\right| \\
& =q^{2(d+1) \operatorname{deg}\left(Q_{i}\right)}-\left(\left(q^{\operatorname{deg}\left(Q_{i}\right)}-1\right) q^{(d-1) \operatorname{deg}\left(Q_{i}\right)}\left(q^{2 \operatorname{deg}\left(Q_{i}\right)}-q^{\operatorname{deg}\left(Q_{i}\right)}\right)\right) \\
& =q^{2(d+1) \operatorname{deg}\left(Q_{i}\right)}\left(1-\frac{q^{2 \operatorname{deg}\left(Q_{i}\right)}-2 q^{\operatorname{deg}\left(Q_{i}\right)}+1}{q^{(d+2) \operatorname{deg}\left(Q_{i}\right)}}\right) \\
& =q^{2(d+1) \operatorname{deg}\left(Q_{i}\right)}\left(1-\frac{\left(q^{\operatorname{deg}\left(Q_{i}\right)}-1\right)^{2}}{q^{(d+2) \operatorname{deg}\left(Q_{i}\right)}}\right) .
\end{aligned}
$$

Therefore for $D$ of degree large enough

$$
\begin{aligned}
\frac{\left|\overline{\mathcal{N}}_{t}^{+} \cap \mathcal{L}(D)^{d+1}\right|}{\left|\mathcal{L}(D)^{d+1}\right|} & =\frac{q^{(d+1)\left(\operatorname{deg}(D)-2 \sum_{i=1}^{t} \operatorname{deg}\left(Q_{i}\right)+1-g\right)}}{q^{(d+1)(\operatorname{deg}(D)+1-g)}} \cdot \prod_{i=1}^{t} q^{2(d+1) \operatorname{deg}\left(Q_{i}\right)}\left(1-\frac{\left(q^{\operatorname{deg}\left(Q_{i}\right)}-1\right)^{2}}{q^{(d+2) \operatorname{deg}\left(Q_{i}\right)}}\right) \\
& =\prod_{i=1}^{t}\left(1-\frac{\left(q^{\operatorname{deg}\left(Q_{i}\right)}-1\right)^{2}}{q^{(d+2) \operatorname{deg}\left(Q_{i}\right)}}\right) .
\end{aligned}
$$

Hence

$$
\mathbb{D}\left(\overline{\mathcal{N}}_{t}^{+}\right)=\lim _{D \in \mathcal{D}} \frac{\left|\overline{\mathcal{N}}_{t}^{+} \cap \mathcal{L}(D)^{d+1}\right|}{\left|\mathcal{L}(D)^{d+1}\right|}=\prod_{i=1}^{t}\left(1-\frac{\left(q^{\operatorname{deg}\left(Q_{i}\right)}-1\right)^{2}}{q^{(d+2) \operatorname{deg}\left(Q_{i}\right)}}\right)
$$


Theorem 7. The density of the set of Eisenstein polynomials with coefficients in $H$ is

$$
\mathbb{D}\left(\mathcal{E}^{+}\right)=1-\prod_{Q \in \mathcal{S}}\left(1-\frac{\left(q^{\operatorname{deg}(Q)}-1\right)^{2}}{q^{(d+2) \operatorname{deg}(Q)}}\right) .
$$

Proof. Again by lemma 4 we have to show that $\lim _{t \rightarrow \infty} \overline{\mathbb{D}}\left(\overline{\mathcal{N}}_{t}^{+} \backslash \mathcal{N}^{+}\right)=0$.

Observe that $\mathcal{E}_{r}^{+} \cap \mathcal{L}(D)^{d+1} \subseteq Q_{r}^{d} \times \mathcal{L}(D)$. We get

$$
\begin{aligned}
& \overline{\mathbb{D}}\left(\overline{\mathcal{N}}_{t}^{+} \backslash \mathcal{N}^{+}\right)=\limsup _{D \in \mathcal{D}} \frac{\left|\left(\overline{\mathcal{N}}_{t}^{+} \backslash \mathcal{N}^{+}\right) \cap \mathcal{L}(D)^{d+1}\right|}{\left|\mathcal{L}(D)^{d+1}\right|} \\
& \leq \limsup _{D \in \mathcal{D}}\left|\bigcup_{\substack{r>t \\
\operatorname{deg}\left(Q_{r}\right) \leq \operatorname{deg}(D)}} \mathcal{E}_{r}^{+} \cap \mathcal{L}(D)^{d+1}\right| q^{-(d+1) \ell(D)} \\
& \leq \limsup _{D \in \mathcal{D}}\left|\bigcup_{\substack{r>t \\
\operatorname{deg}\left(Q_{r}\right) \leq \operatorname{deg}(D)}}\left(Q_{r}^{d} \times \mathcal{L}(D)\right) \cap \mathcal{L}(D)^{d+1}\right| q^{-(d+1) \ell(D)} \\
& \leq \limsup _{D \in \mathcal{D}} \sum_{\substack{r>t \\
\operatorname{deg}\left(Q_{r}\right) \leq \operatorname{deg}(D)}} \frac{\left|\left(Q_{r}^{d} \times \mathcal{L}(D)\right) \cap \mathcal{L}(D)^{d+1}\right|}{q^{(d+1) \ell(D)}} \\
& =\limsup _{D \in \mathcal{D}} \sum_{\substack{r>t \\
\operatorname{deg}\left(Q_{r}\right) \leq \operatorname{deg}(D)}} \frac{\left|Q_{r} \cap \mathcal{L}(D)\right|^{d}|\mathcal{L}(D)|}{q^{(d+1) \ell(D)}} \\
& =\limsup _{D \in \mathcal{D}} \sum_{\substack{r>t \\
\operatorname{deg}\left(Q_{r}\right) \leq \operatorname{deg}(D)}} \frac{\left|Q_{r} \cap \mathcal{L}(D)\right|^{d}}{q^{\operatorname{d\ell }(D)}}
\end{aligned}
$$

which is equation (3). Hence for $t$ going to infinity we obtain $\overline{\mathbb{D}}\left(\overline{\mathcal{N}}_{t}^{+} \backslash \mathcal{N}^{+}\right)=0$.

We now apply Lemma 4 with $n=d+1, A_{t}=\overline{\mathcal{N}}_{t}^{+}$and $A=\mathcal{N}^{+}$obtaining

$$
\mathbb{D}\left(\mathcal{N}^{+}\right)=\lim _{t \rightarrow \infty} \mathbb{D}\left(\overline{\mathcal{N}}_{t}^{+}\right)=\lim _{t \rightarrow \infty} \prod_{i=1}^{t}\left(1-\frac{\left(q^{\operatorname{deg}\left(Q_{i}\right)}-1\right)^{2}}{q^{(d+2) \operatorname{deg}\left(Q_{i}\right)}}\right)=\prod_{Q \in \mathcal{S}}\left(1-\frac{\left(q^{\operatorname{deg}(Q)}-1\right)^{2}}{q^{(d+2) \operatorname{deg}(Q)}}\right) .
$$

We now take the complement

$$
\mathbb{D}\left(\mathcal{E}^{+}\right)=1-\mathbb{D}\left(\mathcal{N}^{+}\right)=1-\prod_{Q \in \mathcal{S}}\left(1-\frac{\left(q^{\operatorname{deg}(Q)}-1\right)^{2}}{q^{(d+2) \operatorname{deg}(Q)}}\right) .
$$

\section{REFERENCES}

[1] Artūras Dubickas. Polynomials irreducible by eisenstein's criterion. Applicable Algebra in Engineering, Communication and Computing, 14(2):127-132, 2003.

[2] X. Guo and G. Yang. The probability of rectangular unimodular matrices over $\mathbb{F}_{q}[x]$. Linear Algebra and its Applications, 438(6):2675-2682, 2013. 
[3] Randell Heyman and Igor E Shparlinski. On the number of eisenstein polynomials of bounded height. Applicable Algebra in Engineering, Communication and Computing, 24(2):149-156, 2013.

[4] J. L. Kelley. General topology. New York: Van Nostrand, 1955.

[5] Giacomo Micheli and Reto Schnyder. On the density of coprime m-tuples over holomorphy rings. To appear in International Journal of Number Theory, 2014. URL arXiv: 1411.6876.

[6] H. Stichtenoth. Algebraic function fields and codes, volume 254. Springer, 2009.

[7] H. Sugita and S. Takanobu. The probability of two $\mathbb{F}_{q}[x]$-polynomials to be coprime. Probability and number theory, Advanced Studies in Pure Mathematics, 49:455-478, 2007.

Institute of Mathematics, University of Zurich, Winterthurerstrasse 190, 8057 Zurich, SWITZERLAND,

E-mail address: edoardo.dotti@uzh.ch

Institute of Mathematics, University of Zurich, Winterthurerstrasse 190, 8057 Zurich, SWITZERLAND,

E-mail address: giacomo.micheli@math.uzh.ch 